\title{
The acute effects of a high-fat and a low-fat meal on cardiac output at rest and during and post exercise in healthy young adults
}

\author{
Victoria G. Rontoyanni and Tom Sanders \\ King's College London, London, UK
}

The effects of postprandial lipaemia on cardiac output (CO) are uncertain. The present study was designed to assess the effect of a meal high in fat $v$. a meal low in fat on $\mathrm{CO}$ at rest and during and post exercise.

A randomised cross-over study design was chosen to compare a high-fat meal (HFM) with an isovolumic and isoenergetic low-fathigh-carbohydrate meal (HCM). Sixty-six subjects were screened for the purposes of the study. Thirty (fifteen females, fifteen males) healthy non-smoking non-obese 18-45-year-old subjects completed the study. Their mean age and BMI were 26.5 (SD 4.9) years and 22.5 (SD 2.8$) \mathrm{kg} / \mathrm{m}^{2}$ respectively. Subjects were allocated to one of two treatment sequences in random order. Measurements of CO were made at 0 (fasting), 60, 120 and 180 min following each test meal, during $12 \mathrm{~min}$ incremental exercise in the postprandial state and at 15,30 and 45 min post exercise. Blood samples for plasma TAG and NEFA were collected in the fasting state, at 180 min postprandially and immediately after exercise. The $12 \mathrm{~min}$ exercise protocol used was cycling at $60 \mathrm{rev} / \mathrm{min}$ and a work rate increased by $25 \mathrm{~W}$ in $3 \mathrm{~min}$ intervals starting at $25 \mathrm{~W}$. A novel re-breathing technique was used to assess CO (InnocorTM; Innovision A/S, Odense, Denmark). Replicate measures indicated that the within-subject CV was $<10 \%$ for measurement of CO. Postprandial differences in means or mean changes were analysed using mixed-design repeated-measures ANOVA. The results are shown in Figs. 1 and 2.

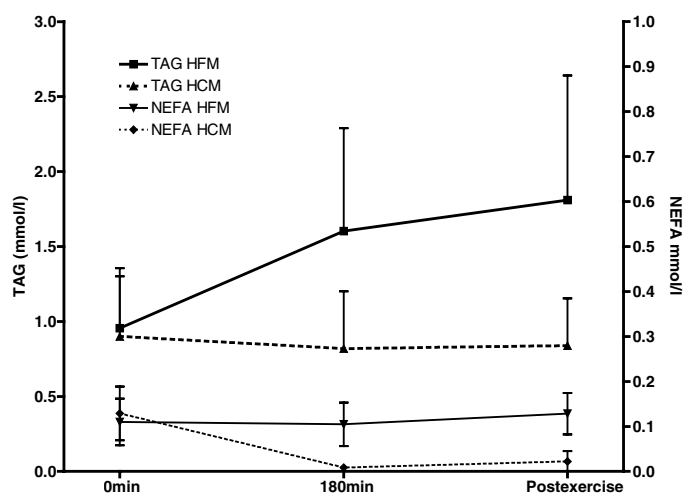

Figure 1. TAG \& NEFA concentrations at fasting, $180 \mathrm{~min}$ postprandially \& postexercise. Values are means with SD.

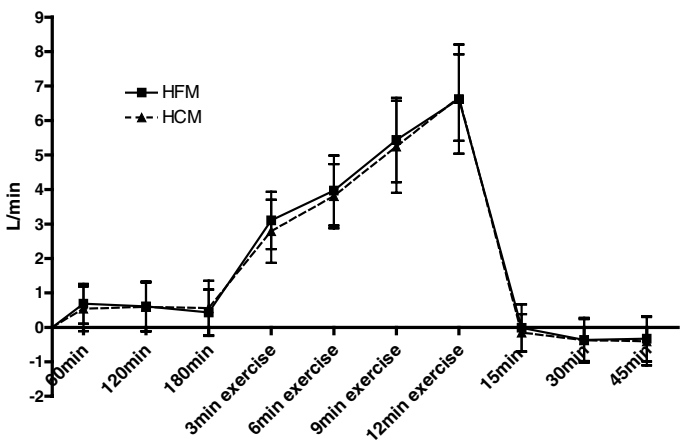

Figure 2. Cardiac output changes at rest, during \& postexercise. Values are mean changes with SD. For $60 \mathrm{~min}, 120 \mathrm{~min}, 180 \mathrm{~min}$ rest, changes are from fasting values. For exercise and postexercise, changes are from 180 min values.

TAG were significantly raised $(P<0.001)$ after the HFM and plasma NEFA were suppressed following the HCM $(P<0.001$; Fig. 1$)$. Fasting plasma NEFA were significantly higher in females $(P=0.002)$ and resulted in a greater decrease $3 \mathrm{~h}$ after the HCM compared with males $(P=0.002)$. The mean $\mathrm{CO}$ at baseline was 5.08 (SD 1.07$) 1 / \mathrm{min}$. There was no meal effect, meal $\times$ time effect or meal $\times$ time $\times$ gender interaction in relation to $\mathrm{CO}$ changes at rest, during exercise or post exercise (Fig. 2). There were some differences in the changes in the components of $\mathrm{CO}$ between males and females. Females showed a higher increase in heart rate compared with males $(P<0.05)$ whereas males showed a greater increase in stroke volume compared with females $(P<0.001)$.

These results show that postprandial lipaemia does not adversely affect resting $\mathrm{CO}$ or CO during exercise in healthy young subjects. In males $\mathrm{CO}$ was increased mainly by an increase in stroke volume whereas in females the increase was mainly in heart rate. 